International Journal of Modern Physics D

(C) World Scientific Publishing Company

\title{
CHAMELEON COSMOLOGY MODEL DESCRIBING THE PHANTOM DIVIDE LINE CROSSING
}

\author{
FRANCESCO CANNATA \\ INFN, sezione di Bologna, Via Irnerio 46, 40126 Bologna, Italy \\ cannata@bo.infn.it \\ ALEXANDER Yu KAMENSHCHIK \\ Dipartimento di Fisica and INFN, sezione di Bologna, Via Irnerio 46, 40126 Bologna,Italy \\ L.D. Landau Institute for Theoretical Physics of the Russian Academy of Sciences, Kosygin \\ str. 2, 119334 Moscow, Russia \\ kamenshchik@bo.infn.it
}

\begin{abstract}
An exact solution describing the evolution of the type Bang-to-Rip with the phantom divide line crossing is constructed in the Chameleon cosmology model, based on two independent functions of the scalar field.
\end{abstract}

Keywords: dark energy; chameleon cosmology; phantom divide line crossing

\section{Introduction}

The discovery of cosmic acceleration 1 has stimulated an intensive study of models of dark energy 2 responsible for the origin of this phenomenon. Dark energy is characterized by a negative pressure whose relation to the energy density $w=p / \varepsilon$ is less than $-1 / 3$. Moreover, if this relation happens to be less than -1 such kind of dark energy is called "phantom" dark energy 3 . The breakdown of the condition $w>-1$ implies the so called super-acceleration of cosmological evolution, which in some models culminates approaching a new type of cosmological singularity called Big Rip 4 . While the cosmological constant $(w=-1)$ is still a possible candidate for the role of dark energy, there are observations giving indications in favor of the models where the equation of state parameter $w$ not only changes with time, but could become less than $-1 \mathrm{no}$. There are also observations 6 indicating the phenomenon of the phantom divide line crossing 7 , when the parameter $w$ changes between the phantom and non-phantom phases. A standard way to introduce the phantom energy is to consider a scalar field with the negative sign of the kinetic energy term, usually called "phantom" scalar field. The simplest way of describing the phenomenon of the phantom divide line crossing is to introduce two scalar fields - phantom and non-phantom as in the so called quintom models 8 . Some other models are based on the use of the PT symmetry ideas 9|10|11. Note that the phantom divide line crossing can occur also in models based on one scalar field if some special 
potential are chosen 12 or if the non-minimal coupling between a scalar field and gravity is included 13 .

Naturally, consideration of two scalar fields open wider opportunities for description of any prescribed cosmological evolution, than working with a single field 14 .

Recently, another class of cosmological dark energy models was put forward - the so called chameleon cosmology models 15. In this models the scalar field interacts with some kind of matter, behaving as perfect fluid. The Lagrangian of the model contains a term, where the matter interacts with the effective metric (physical metric, multiplied by a conformal factor depending on the scalar field). The energy density of the perfect fluid is proportional to the trace of its energy-momentum tensor, multiplied by this conformal factor. On the other hand the effective mass of the scalar field becomes variable, depending on the energy density of matter, which changes in the process of the cosmological evolution.

Lately, an alternative version of the Chameleon cosmology was proposed in paper 16. Here the term, describing the interaction between the chameleon scalar field and matter has a very general multiplicative form and is equal to some arbitrary function of the scalar field multiplied by the Lagrangian of matter, which on the Friedmann-Robertson-Walker background coincides with the pressure of the matter. Thus, one has two arbitrary functions of the scalar field to play with - the standard potential and the potential-like function describing the interaction of matter with the chameleon field. Choosing properly these two functions of the scalar field one can describe different kinds of cosmological evolution, including those where the universe undergoes the phantom divide line crossing. The authors of paper 16 use the numerical integration of the corresponding equations of motion and compare the results with the data coming from time evolution of the cosmological redshift of distant sources.

We remark that having two functions of a scalar field one gets a sufficient freedom to construct exactly solvable solutions of the cosmological equations, giving some prescribed cosmological evolution. Just like choosing exponential potentials one can find exact solutions desribing power-law expansion of the universe (see e.g. 17), here one can try to construct two potential-like functions providing the existence of exact cosmological solutions, undergoing the phantom divide line crossing. In fact, this is the task of the present paper.

We consider the flat Friedmann model with the metric

$$
d s^{2}=d t^{2}-a^{2}(t) d l^{2} .
$$

The Hubble parameter behaves as

$$
H(t) \equiv \frac{\dot{a}(t)}{a(t)}=\frac{\alpha t_{R}}{t\left(t_{R}-t\right)},
$$

where $\alpha$ is some positive parameter of the order 1 and "dot" means as usual the derivative with respect to the cosmic time $t$. Such a cosmological evolution was 
considered in papers $10[14$ in the the context of two-field models and was called "Bang-to-Rip" evolution. Here the universe begins its evolution from the Big Bang singularity when the Hubble parameter behaves as $H(t) \sim \frac{\alpha}{t}$ and ends in the Big Rip singularity at the moment $t=t_{R}$. At the moment $t=\frac{t_{R}}{2}$ the phantom divide line is crossed by the universe. Indeed, the phantom divide line crossing means that the relation between the pressure and the energy density becomes equal to -1 . That means that in the energy conservation law $\dot{\varepsilon}=-3 H(\varepsilon+p)$ the right-hand side vanishes, and so does the time derivative of the total energy density in its left-hand side. The energy density is proportinal in the flat Friedmann model to the Hubble parameter squared. Hence, the time derivative of the Hubble parameter $H$ should vanish too. The time derivative of the Hubble parameter from Eq. (2) is

$$
\dot{H}=\frac{2 \alpha t_{R}\left(2 t-t_{R}\right)}{t^{2}\left(t_{R}-t\right)^{2}}
$$

and vanishes at $t=t_{R} / 2$.

We shall consider the minimally coupled to gravity scalar field $\phi$ with the potential $V(\phi)$ whose interaction with the perfect fluid is described by the term in the Lagrangian, which on the Friedmann background looks like 16 :

$$
L_{\text {scalar }+ \text { matter }}=\gamma \rho f(\phi),
$$

where the coefficient $\gamma$ relates the energy density $\rho$ and the pressure $p$ of matter:

$$
p=\gamma \rho,
$$

and $f(\phi)$ is some function of the scalar field $\phi$. In the next section we shall find the form of the functions $V(\phi)$ and $f(\phi)$, and the exact solution for $a(t)$ and $\phi(t)$ matching the evolution (2). In the third section we discuss the bounds on the parameters of the model. The last section contains some concluding remarks.

\section{An exactly solvable Chameleon field cosmological model}

We shall fix the fundamental constants in such a way to give to the Friedmann equation a particularly simple form:

$$
H^{2}=\varepsilon,
$$

where $\varepsilon$ is the total energy density of the scalar field and matter. On the flat Friedmann background this total energy density is

$$
\varepsilon=\frac{\dot{\phi}^{2}}{2}+V(\phi)+\rho f(\phi) .
$$

The Klein-Gordon equation for the scalar field $\phi$ is

$$
\ddot{\phi}+3 H \dot{\phi}+V^{\prime}(\phi)+\gamma \rho f^{\prime}(\phi)=0,
$$

where "prime" stays for the derivative with respect to $\phi$. The total energy density $\varepsilon$ satisfies the energy conservation law

$$
\dot{\varepsilon}+3 H(\varepsilon+P)=0,
$$


where $P$ is the total pressure of the matter and of the scalar field which is equal to

$$
P=\gamma \rho+\frac{\dot{\phi}^{2}}{2}-V(\phi)
$$

Substituting Eqs. (7) and (10) into Eq. (9) we obtain

$$
\dot{\phi}\left(\ddot{\phi}+3 H \dot{\phi}+V^{\prime}+\rho f^{\prime}\right)=-3(1+\gamma) H \rho f-\dot{\rho} f .
$$

Using Eq. (8) we can simplify the left-hand side of Eq. (11) coming to

$$
(1-\gamma) \rho \dot{f}+3(1+\gamma) H \rho f+\dot{\rho} f=0 .
$$

Integrating Eq. (12) one obtains for the energy density of the matter

$$
\rho(t)=\frac{\rho_{0}}{f^{1-\gamma}(\phi(t)) a^{3(1+\gamma)}(t)},
$$

where $\rho_{0}$ is an integration constant.

Now the Friedmann and Klein-Gordon equations can be rewritten as

$$
\begin{gathered}
H^{2}=\frac{\dot{\phi}^{2}}{2}+V+\frac{\rho_{0}}{f^{1-\gamma} a^{3(1+\gamma)}}, \\
\ddot{\phi}+3 H \dot{\phi}+V^{\prime}+\frac{\gamma \rho_{0} f^{\prime}}{f^{1-\gamma} a^{3(1+\gamma)}}=0 .
\end{gathered}
$$

We have chosen the cosmological evolution described by the Hubble variable (2). Correspondingly the cosmological radius $a(t)$ is

$$
a(t)=\frac{a_{0} t^{\alpha}}{\left(t_{R}-t\right)^{\alpha}},
$$

where $a_{0}$ is a positive constant. Now we can rewrite the matter energy density $\rho(t)$ as

$$
\rho(t)=\frac{M\left(t_{R}-t\right)^{3 \alpha(1+\gamma)}}{f^{1-\gamma} t^{3 \alpha(1+\gamma)}},
$$

where we have introduced a new constant

$$
M=\frac{\rho_{0}}{a_{0}^{3(1+\gamma)}} .
$$

The term $\rho f$ in the right-hand side of the Friedmann equation (14) now looks as

$$
\rho f=\frac{M f^{\gamma}\left(t_{R}-t\right)^{3 \alpha(1+\gamma)}}{t^{3 \alpha(1+\gamma)}} .
$$

Now, the left-hand side of Eq. (14) behaves as $\sim 1 / t^{2}\left(t_{R}-t\right)^{2}$. If we would like the contribution of the kinetic term of the scalar field $\dot{\phi}^{2} / 2$ to have the same structure we should require that the time dependence of the scalar field is

$$
\phi(t)=\phi_{0} \ln \frac{t}{t_{R}-t},
$$


where $\phi_{0}$ is a constant to be determined. Then, we shall look for the time dependence of the potential $V$ in the form

$$
V=\frac{V_{0}+V_{1} t}{t^{2}\left(t_{R}-t\right)^{2}}
$$

We shall try to find time dependence of the function $f$ from the relation

$$
f^{\gamma}=\left(f_{0}+f_{1} t\right) t^{3 \alpha(1+\gamma)-2}\left(t_{R}-t\right)^{-3 \alpha(1+\gamma)-2} .
$$

Substituting Eqs. (22), (20), (21) and (22) into Eq. (14) we obtain the following constraints on the constants :

$$
\begin{gathered}
\alpha^{2} t_{R}^{2}=M f_{0}+\frac{\phi_{0}^{2} t_{R}^{2}}{2}+V_{0}, \\
V_{1}+M f_{1}=0 .
\end{gathered}
$$

Now, let us consider the Klein-Gordon equation (15). The second time derivative of the scalar field is

$$
\ddot{\phi}=-\frac{\phi_{0}}{t^{2}}+\frac{\phi_{0}}{\left(t_{R}-t\right)^{2}}=\frac{\phi_{0} t_{R}\left(2 t-t_{R}\right)}{t^{2}\left(t_{R}-t\right)^{2}} .
$$

The "friction" term is

$$
3 H \dot{\phi}=\frac{3 \alpha \phi_{0} t_{R}^{2}}{t^{2}\left(t_{R}-t\right)^{2}} .
$$

The derivative of the potential is

$$
V^{\prime}(\phi)=\frac{-2 V_{0} t_{R}+\left(4 V_{0}-V_{1} t_{R}\right) t+3 V_{1} t^{2}}{\phi_{0} t_{R} t^{2}\left(t_{R}-t\right)^{2}} .
$$

and the term, including the interaction with matter is

$$
\begin{aligned}
& \gamma f^{\prime} \rho=\frac{1}{\phi_{0} t_{R} t^{2}\left(t_{R}-t\right)^{2}} \times\left(f_{0} t_{R}(3 \alpha(1+\gamma)-2)\right. \\
& +\left(4 f_{0}+f_{1} t_{R}(3 \alpha(1+\gamma)-1)+3 f_{1} t^{2}\right) .
\end{aligned}
$$

Now, substituting the expressions (15)-(28) into Eq. (15) we obtain the following constraints:

$$
\begin{gathered}
-\phi_{0}^{2} t_{R}^{2}+3 \phi_{0}^{2} t_{R}^{2} \alpha-2 V_{0}+M f_{0}(3 \alpha(1+\gamma)-2)=0, \\
2 \phi_{0}^{2} t_{R}^{2}+4 V_{0}-V_{1} t_{R}+4 M f_{0}+M f_{1} t_{R}(3 \alpha(1+\gamma)-1)=0, \\
3 V_{1}+3 M f_{1}=0 .
\end{gathered}
$$

The last condition (31) coincides with (24).

Now we can solve the constraints and find the relations between the parameters. We shall choose as free parameters the amplitude of the scalar field $\phi_{0}$ and the "quantity of matter" $M>0$. From Eqs. (23) and (30) we find

$$
V_{1}=\frac{4 \alpha t_{R}}{3(1+\gamma)} \text {. }
$$


Then from Eq. (24) follows

$$
f_{1}=-\frac{4 \alpha t_{R}}{3 M(1+\gamma)}
$$

From Eqs. (23) and (29) we find

$$
f_{0}=\frac{t_{R}^{2}\left(2 \alpha-\phi_{)}^{2}\right)}{3 M(1+\gamma)}
$$

and

$$
V_{0}=\frac{t_{R}^{2}\left(3 \alpha(3 \alpha(1+\gamma)-2)+3 \phi_{0}^{2}(1-\gamma)\right)}{6(1+\gamma)} .
$$

We should also now express the time parameter $t$ in terms of the scalar field $\phi$. It follows immediately from Eq. (20) that

$$
t=\frac{t_{R}}{1+\exp \left(-\frac{\phi}{\phi_{0}}\right)} \text {. }
$$

Substituting the parameters $V_{0}, V_{1}, f_{0}$ and $f_{1}$ from Eqs. (32)-(V0) and the expression for the time parameter $t$ from Eq. (36) into Eqs. (21) and (22) we find the explicit expressions for the potential $V(\phi)$ and the function $f(\phi)$ :

$$
\begin{gathered}
V(\phi)=\frac{8 \cosh ^{4} \frac{\phi}{2 \phi_{0}}}{3(1+\gamma)}\left(6 \alpha^{2}(1+\gamma)+3 \phi_{0}^{2}(1-\gamma)+4 \alpha \tanh \frac{\phi}{2 \phi_{0}}\right), \\
f(\phi)=\left(-\frac{16 \cosh ^{4} \frac{\phi}{2 \phi_{0}} \exp \left(3 \alpha(1+\gamma) \frac{\phi}{\phi_{0}}\right)}{3 M t_{R}^{2}(1+\gamma)}\left(3 \phi_{0}^{2}+2 \alpha \tanh \frac{\phi}{2 \phi_{0}}\right)\right)^{\frac{1}{\gamma}} .
\end{gathered}
$$

\section{Parameters of the model}

The parameters $\alpha$ and $t_{R}$ are given by the cosmological dynamics of the model (2). The parameter $\phi_{0}$ is free. Without loosing generality we can choose it positive. Correspondingly the value of the scalar field $\phi$ (see Eq. (20) ) runs from -infty at the Big Bang beginning of the cosmological evolution to $+\infty$ at its end at the moment $t=t_{R}$ when it encounters the Big Rip singularity. It crosses the zero value at $t=t_{R} / 2$, i.e. at the moment of the phantom divide line crossing. Now, looking at the expression for the function $f(\phi)$ describing the interaction between the chameleon scalar field and the matter, we see that it is well defined only if the expression, which should be elevated into the power $1 / \gamma$ is always positive, or if the parameter $\gamma$ satisfies some additional restrictions. Thus, we can consider two cases.

The case I. The expression in the bracket in Eq. (38) is always positive. It is possible if and only if

$$
\phi_{0} \geq \sqrt{\frac{2 \alpha}{3}}
$$




$$
\gamma<-1
$$

Then if the parameter $\alpha>\frac{1}{3}$ and

$$
\phi_{0}>\sqrt{\frac{4 \alpha-6 \alpha^{2}(1+\gamma)}{3(1-\gamma)}}
$$

the potential $V(\phi)$ is always negative. If $\alpha>\frac{1}{3}$ and

$$
\sqrt{\frac{2 \alpha}{3}}<\phi_{0}<\sqrt{\frac{4 \alpha-6 \alpha^{2}(1+\gamma)}{3(1-\gamma)}}
$$

the potential $V(\phi)$ changes sign at

$$
\phi=2 \phi_{0} \operatorname{arctanh} \frac{5 \alpha^{2}(1+\gamma)+3 \phi_{0}^{2}(1-\gamma)}{4 \alpha} .
$$

If $\alpha<\frac{1}{3}$ the potential is always negative.

The case II. If at least one of two inequalities (39), (40) is broken the expression for $f^{\gamma}$ in Eq. (38) cannot be always nonnegative. Hence, we should impose a following condition on the factor $\gamma$ :

$$
\gamma=\frac{2 m+1}{n}
$$

where $m$ and $n$ are integers. In this case the expression for $f$ is well defined. The sign of the potential depends on the interplay of three parameters $\phi_{0}, \gamma$ and $\alpha$.

\section{Conclusion}

We have constructed an exact solution for a particular Chameleon cosmological model of the type considered in paper 16. The universe in this solution begins its evolution from Big Bang singularity, undergoes a phantom divide line crossing and ends in the Big Rip singularity. The two potential-like functions of the chameleon scalar field have a rather simple analytic form. Note that this form is simpler than the potential functions in two-scalar model, providing the same cosmological evolu-

tion 10. Thus, we hope that our version of the Chameleon model can be useful for the analysis of the phantom divide line crossing phenomenon.

\section{Acknowledgments}

This work was partially supported by Grants RFBR 08-02-00923 and LSS4899.2008.2.

\section{References}

1. A. G. Riess et al. [Supernova Search Team Collaboration], Astron. J. 116 (1998) 1009; S. Perlmutter et al. [Supernova Cosmology Project Collaboration], Astrophys. J. 517 (1999) 565. 
2. V. Sahni and A. A. Starobinsky, Int. J. Mod. Phys. D 9 (2000) 373;

V. Sahni and A. A. Starobinsky, Int. J. Mod. Phys. D 15 (2006) 2105.

T. Padmanabhan, Phys. Rept. 380 (2003) 235;

P. J. E. Peebles and B. Ratra, Rev. Mod. Phys. 75 (2003) 559;

E. J. Copeland, M. Sami and S. Tsujikawa, Int. J. Mod. Phys. D 15 (2006) 1753.

3. R. R. Caldwell, Phys. Lett. B 545 (2002) 23.

4. A. A. Starobinsky, Grav. Cosmol. 6 (2000) 157;

R. R. Caldwell, M. Kamionkowski and N. N. Weinberg, Phys. Rev. Lett. 91 (2003) 071301.

5. U. Alam, V. Sahni, T. D. Saini and A. A. Starobinsky, Mon. Not. Roy. Astron. Soc. 354 (2004) 275.

6. V. Barger, Y. Gao and D. Marfatia, Phys. Lett. B 648 (2007) 127;

A. Gong and A. Wang, Phys. Rev. D 75 (2007) 0435520;

U. Alam, V. Sahni and A.A. Starobinsky, JCAP 0702 (2007)011;

S. Nesseris and L. Perivolaropoulos, JCAP 0702 (2007) 025;

G. B. Zhao, J. Q. Xia, H. Li et al, Phys. Lett. B 648 (2007) 8;

P. Serra, A. Heavens and A. Melchiorri, MNRAS 3791 (2007) 169;

T.M. Davis, E. Mortsell, J. Sollerman et al., Astrophys. J. 666 (2007) 716;

E. l. Wright, Astrophys. J. 664 (2007) 633;

Y. Wang and P. Mukherjee, Phys. Rev. D 76 (2007) 103533.

7. B. Boisseau, G. Esposito-Farese, D. Polarski and A.A. Starobinsky, Phys. Rev. Lett. 85 (2000) 2236;

G. Esposito-Farese and D. Polarski, Phys. Rev. D 63 (2001) 063504;

A. Vikman, Phys. Rev. D 71 (2005) 023515;

L. Perivolaropoulos, Phys. Rev. D 71 (2005) 063503;

B. McInnes, Nucl. Phys. B 718 (2005) 55;

I. Ya. Aref'eva, A.S. Koshelev and S. Yu.Vernov, Phys. Rev. D 72 (2005) 064017;

L. Perivolaroupoulos, JCAP 0510 (2005) 001;

R.R. Caldwell and M. Doran, Phys. Rev. D 72 (2005) 043527;

V. Sahni and Yu. Shtanov, JCAP 0311 (2003) 014;

V. Sahni and L. Wang, Phys. Rev. D 62 (2000) 103517;

H. Wei and R.G. Cai, Phys. Rev. D 72 (2005) 123507;

H. Wei and R.G. Cai, Phys. Lett. B 634 (2006) 9;

R.G. Cai and A. Wang, JCAP 0503 (2005) 002.

8. X. L. Feng, X. L. Wang and X. M. Zhang, Phys. Lett. B 607 (2005) 35;

L. Perivolaropoulos, Phys. Rev. D 71 (2005) 063503;

G. B. Zhao, J. Q. Xia, M. Li, B. Feng and X. Zhang, Phys. Rev. D 72123515 (2005);

J. Q. Xia, B. Feng and X. M. Zhang, Mod. Phys. Lett. A 202409 (2005);

B. Feng, M. Li, Y. S. Piao and X. Zhang, Phys. Lett. B 634101 (2006);

Z. K. Guo, Y. S. Piao, X. M. Zhang and Y. Z. Zhang, Phys. Lett. B 608177 (2005);

H. Wei, R. G. Cai and D. F. Zeng, Class. Quant. Grav. 223189 (2005);

R. Lazkoz and G. Leon, Phys. Lett. B 638303 (2006);

X. F. Zhang and T. Qiu, Phys. Lett. B 642187 (2006);

W. Zhao, Phys. Rev. D 73123509 (2006);

M. Alimohammadi and H. M. Sadjadi, Phys. Lett. B 648113 (2007);

Z. K. Guo, Y. S. Piao, X. Zhang and Y. Z. Zhang, Phys. Rev. D 74127304 (2006);

Y. f. Cai, H. Li, Y. S. Piao and X. m. Zhang, Phys. Lett. B 646141 (2007);

X. Zhang, Phys. Rev. D 74103505 (2006);

M. R. Setare, Phys. Lett. B 641130 (2006);

Y. f. Cai, M. z. Li, J. X. Lu, Y. S. Piao, T. t. Qiu and X. m. Zhang, Phys. Lett. B 
6511 (2007);

R. Lazkoz, G. Leon and I. Quiros, Phys. Lett. B 649103 (2007);

M. Alimohammadi, Gen. Rel. Grav. 40107 (2008);

M. R. Setare and E. N. Saridakis, Phys. Lett. B 668177 (2008);

J. Sadeghi, M. R. Setare, A. Banijamali and F. Milani, Phys. Lett. B 66292 (2008);

H. H. Xiong, Y. F. Cai, T. Qiu, Y. S. Piao and X. Zhang, Phys. Lett. B 666212 (2008);

Y. F. Cai and J. Wang, Class. Quant. Grav. 25165014 (2008);

S. Zhang and B. Chen, Phys. Lett. B 6694 (2008);

M. R. Setare and E. N. Saridakis, Int. J. Mod. Phys. D 18549 (2009);

M. R. Setare and E. N. Saridakis, JCAP 0809026 (2008);

M. R. Setare and E. N. Saridakis, Phys. Lett. B 671331 (2009);

K. Nozari, M. R. Setare, T. Azizi and N. Behrouz, Phys. Scripta 80025901 (2009);

M. R. Setare and E. N. Saridakis, Phys. Rev. D 79043005 (2009);

L. P. Chimento, M. Forte, R. Lazkoz and M. G. Richarte, Phys. Rev. D 79043502 (2009);

E. N. Saridakis, arXiv:0903.3840 [astro-ph.CO];

H. Wei and S. N. Zhang, Phys. Rev. D 76063005 (2007);

Y. F. Cai, E. N. Saridakis, M. R. Setare and J. Q. Xia, arXiv:0909.2776 [hep-th];

H. Zhang, arXiv:0909.3013 [astro-ph.CO].

9. A. A. Andrianov, F. Cannata and A. Y. Kamenshchik, J. Phys. A 39 (2006) 9975;

10. A. A. Andrianov, F. Cannata and A. Y. Kamenshchik, Int. J. Mod. Phys. D 15 (2006) 1299.

11. A. A. Andrianov, F. Cannata, A. Y. Kamenshchik and D. Regoli, Int. J. Mod. Phys. D 19 (2010) 97.

12. A. A. Andrianov, F. Cannata and A. Y. Kamenshchik, Phys. Rev. D 72 (2005) 043531;

F. Cannata and A. Y. Kamenshchik, Int. J. Mod. Phys. D 16 (2007) 1683;

13. R. Gannouji, D. Polarski, A. Ranquet and A. A. Starobinsky, JCAP 0609 (2006) 016.

14. A. A. Andrianov, F. Cannata, A. Y. Kamenshchik and D. Regoli, JCAP 0802 (2008) 015.

15. J. Khoury and A. Weltman, Phys. Rev. Lett. 93 (2004) 171104;

J. Khoury and A. Weltman, Phys. Rev. D 69 (2004) 044026;

P. Brax, C. van de Bruck, A. C. Davis, J. Khoury and A. Weltman, Phys. Rev. D 70 (2004) 123518.

16. H. Farajollahi and A. Salehi, Cosmic Dynamics in the Chameleon Cosmology, arXiv:1004.3508 [gr-qc].

17. F. Lucchin and S. Matarrese, Phys. Rev. D 32 (1985) 1316;

J. J. Halliwell, Phys. Lett. B 185 (1987) 341.

A. B. Burd and J. D. Barrow, Nucl. Phys. B 308 (1988) 929;

V. Gorini, A. Y. Kamenshchik, U. Moschella and V. Pasquier, Phys. Rev. D 69 (2004) 123512 . 